\title{
Chemoprophylaxis for pulmonary aspergillosis during intensive chemotherapy
}

\author{
F Cowie, S T Meller, P Cushing, R Pinkerton
}

\begin{abstract}
Three children who developed pulmonary aspergillosis while being treated for leukaemia or non-Hodgkin's lymphoma. Each child continued with intensive myelosuppressive chemotherapy regimens during the infection and each was successfully treated with antifungal prophylaxis based on itraconazole by mouth. Amphotericin $B$ was also given during periods of severe neutropenia. No reactivation of the fungal infection was seen. (Arch Dis Child 1994; 70: 136-138)
\end{abstract}

Fungal infections in patients with cancer are usually due to opportunistic infections (candida or aspergillus) developing during periods of profound immunosuppression with neutropenia.

Aspergillus is a ubiquitous fungus in most parts of the world. Infection (commonly of the respiratory tract) is acquired by the inhalation of spores. Normal host defences include the pulmonary alveolar macrophages (which inhibit spore germination) and adequate circulating neutrophils (which destroy fungal hyphae). Infection in patients with cancer may be a primary infection or reactivation of a previous focus ${ }^{1}$ and can affect any organ system. Acute invasive aspergillosis is the most common invasive aspergillus infection in immunocompromised patients and may be seen as either haemorrhagic infarction or necrotising bronchopneumonia. Chronic necrotising aspergillosis is a slowly progressive form of invasive aspergillosis and is often associated with previous lung disease. Aspergilloma may develop in the lung (classically in an old tuberculous cavity over several years) or in the upper respiratory tract.

The reactivation rate of fungal pneumonia during subsequent neutropenic episodes is $52 \%$ in adults with acute non-lymphocytic leukaemia. ${ }^{12}$ This risk has often precluded the administration of further intensive chemotherapy regimens with a potentially adverse effect on outcome.

Children's

Department, Royal

Marsden Hospital, Downs Road, Sutton, Surrey SM2 5PT. F Cowie

$S$ T Meller

P Cushing

R Pinkerton

Correspondence to: Dr Pinkerton.

Accepted 28 September 1993 (UKAML) protocol receiving $\mathrm{ADE} 10+3+5$ (cytarabine, doxorubicin, and etoposide) and then $\mathrm{ADE} 8+3+5$. After the recovery of his white cell count after the second course of treatment he developed invasive pulmonary aspergillosis. This was diagnosed by radiography, computed tomography (fig 1), bronchoscopy, and by the demonstration of fungal hyphae on bronchioalveolar lavage.

$\mathrm{He}$ was treated with amphotericin B (nebulised and intravenous $1 \mathrm{mg} / \mathrm{kg}$ ) and itraconazole by mouth ( $70 \mathrm{mg}$ daily). With a computed tomogram documenting an improvement in his lungs, he continued to be treated with itraconazole by mouth alone, was withdrawn from the UKAML trial, and proceeded to autologous bone marrow transplantation with melphalan/total body irradiation. Itraconazole was changed to amphotericin B for the period that he was unable to tolerate drugs by mouth. He continued to be treated with itraconazole until six months after the autograft. He remained free of fungal infection, but relapsed and died of leukaemia two years after autologous bone marrow transplantation.

\section{PATIENT 2}

A 3 year old boy presented with $\mathrm{Ki} 1$ positive large cell anaplastic non-Hodgkin's lymphoma. He received pulsed chemotherapy on the United Kingdom Children's Cancer Study Group (UKCCSG) MACHO protocol (vincristine, cyclophosphamide, doxorubicin, methotrexate, and cytarabine) and his disease responded dramatically to the first cycle. After the eighth course of treatment, while neutropenic, he developed a pulmonary aspergilloma, diagnosed radiologically (fig 2), which was treated with amphotericin B (intravenous $1 \mathrm{mg} / \mathrm{kg}$ ) and parental white blood cell transfusions. His temperature settled with neutrophil recovery (after four days); he then underwent surgical resection (lobectomy).

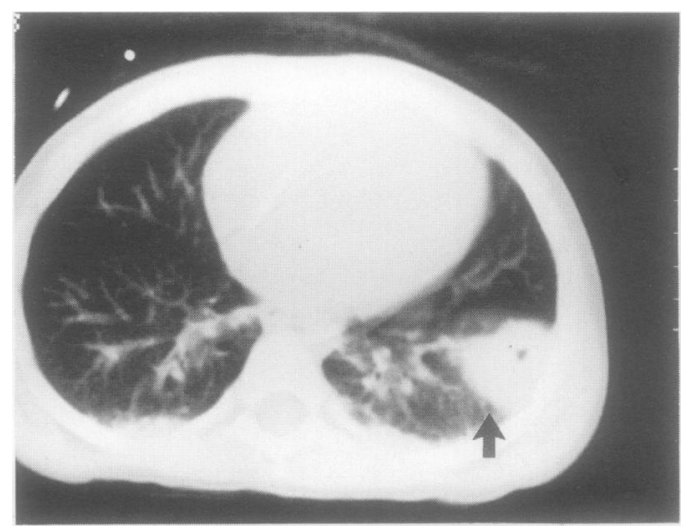

Figure 1 Computed tomogram of the chest of patient 1 showing a large cavitating mass with surrounding interstitial changes, consistent with invasive aspergillosis. 


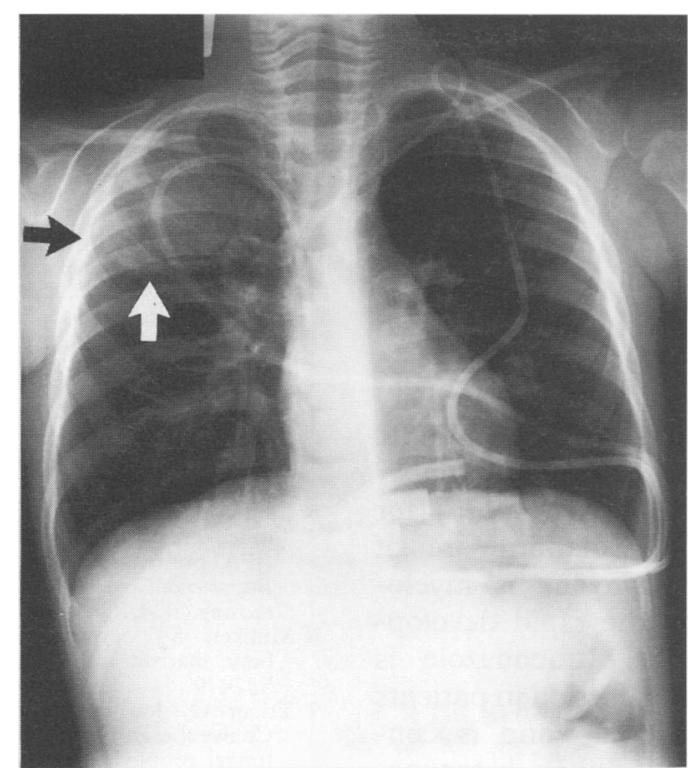

Figure 2 Chest radiograph of patient 2 showing a right upper lobe aspergilloma.

Histology of the resected lobe showed a chronic necrotising aspergillus pneumonia. $\mathrm{He}$ continued to be treated with amphotericin B until the completion of chemotherapy (four further courses) and then received a further two months of treatment with itraconazole by mouth (100 mg/day). He is currently alive and free of disease four years later.

\section{PATIENT 3}

A 3 year old boy presented with acute myeloid leukaemia and was treated on the UKAML 10 protocol. He went into remission with $\mathrm{ADE}$ $10+3+5$ but developed a severe allergy to etoposide; his second course of treatment was DAT $8+3+5$ (daunorubicin, cytarabine, and 6-thioguanine) followed by MACE (amsacrine, cytarabine, and etoposide) without the etoposide. He was then electively autografted with melphalan/total body irradiation. During initial chemotherapy (ADE) he developed an erosive lesion in the left inferior turbinate and the roof of his hard palate. Culture of a piece of necrotic tissue showed aspergillus infection. This was treated with amphotericin B (intravenous $1 \mathrm{mg} / \mathrm{kg}$ ) daily until recovery of his white blood cell count after MAC(E), then on alternate days until a repeat biopsy sample did not show a fungal infection, after which he continued to be treated with itraconazole $(100 \mathrm{mg} /$ day $)$ alone. During the transplant period he received itraconazole daily (replaced by amphotericin B for the neutropenic period) until six months after autologous bone marrow transplantation. $\mathrm{He}$ is currently free of disease and had his palate repaired three years ago.

\section{Discussion}

In immunocompromised patients the incidence of fungal infection is associated with the duration of neutropenia, ${ }^{3-5}$ and damaged tissue (for example, from a previous bacterial infection) probably serves as a nidus for the infection. The resolution of infection is associated with neutrophil recovery, though chronic deep candidiasis has been recognised in patients in remission. $\mathrm{Up}$ to $30 \%$ of patients who die during bone marrow transplantation have evidence of fungal infection at necropsy. ${ }^{6}$ Aggressive use of antifungal drugs (for example, amphotericin B) may induce fungal stasis $^{2}$ until neutrophil recovery.

The incidence of deep fungal infection in patients with leukaemia is $14-40 \% .^{7-11}$ In those undergoing bone marrow transplantation it is $9.5-30 \% .^{3612}$ The results of treating such infection are disappointing, with less than $20 \%$ survival. ${ }^{1213}$

Antifungal drugs with activity against aspergillus $^{14}$ include amphotericin B, flucytosine, ketoconazole, and the triazole derivative itraconazole. Other polyenes (for example, nystatin), imidazoles (clotrimazole and miconazole), and the triazole fluconazole have no useful activity. Data from in vitro sensitivity testing does not correlate with clinical responses to single or multiple drug treatments for fungal infection, however, and the role of antifungal treatment in patients with cancer is to achieve fungal stasis until the granulocytes recover.

Itraconazole is a triazole derivative given by mouth which is less toxic than the other triazoles, ${ }^{14} 15$ and has greater efficacy against aspergillus than ketoconazole or fluconazole, ${ }^{1516}$ and good efficacy against other systemic fungal infections. It acts by inhibiting ergosterol synthesis in fungal cell membranes. Absorption is increased by the presence of food in the stomach and decreased by administration via a nasogastric tube, ${ }^{15}$ or by concomitant $\mathrm{H}_{2}$ antagonists. The dose in adults is $100-200 \mathrm{mg}$ twice a day and 3-5 $\mathrm{mg} / \mathrm{kg} /$ day has been used in children. Steady state kinetics are reached in two weeks, but efficacy relies on adequate blood levels $(>250$ $\mathrm{ng} / \mathrm{ml}$ ), which are reached within 24 hours in non-neutropenic patients receiving $200 \mathrm{mg}$ twice a day..$^{7-9}$ In neutropenic patients these levels are more difficult to achieve, however, and less than $50 \%$ of patients achieve adequate levels in blood, even with an increase in dose to $400 \mathrm{mg}$ twice a day. High levels in tissue are achieved despite low levels in serum, but this does not correlate with effect. ${ }^{14}$ Itraconazole is metabolised by the liver and excreted faecally. Adverse events are minor and generally gastrointestinal, with an occasional mild reversible increase in liver enzymes. The latter was not observed in these patients. In the UK it is currently only licensed for the treatment of superficial infections and is not recommended in children.

Amphotericin B, flucytosine, and ketoconazole have all been used successfully in treating systemic fungal infections, ${ }^{14}$ though aspergillus is known to respond poorly to amphotericin B in the absence of granulocytes. Although there have been no randomised clinical trials comparing the activity of itraconazole with these drugs in aspergillus infection, there are a few case reports and small trials which point to the usefulness of itraconazole. ${ }^{416-18}$ Aspergilloma 
infection is unlikely to be cured by treatment with itraconazole alone, but invasive aspergillosis may have up to a $70 \%$ response rate (though no cures have been reported in patients with continuing neutropenia). ${ }^{14}$

Primary antifungal prophylaxis is generally attempted in patients with cancer; however, there is no single effective drug. Nystatin and amphotericin by mouth are traditionally used and decrease the incidence of systemic candidiasis. ${ }^{6}$ Ketoconazole has poor activity against aspergillus and non-albicans candida, and is difficult to use in allografted patients. ${ }^{6}$ Precautions to decrease exposure to spores (for example, laminar airflow isolation) are of doubtful efficacy. ${ }^{12} 19$ Flucytosine is myelosuppressive and results in the rapid development of fungal resistance. Itraconazole is useful in preventing fungal infection in patients with leukaemia or lymphoma, ${ }^{9}$ and is considered to be more effective than ketoconazole, ${ }^{710}$ though adequate levels in the blood are important.

A previously treated fungal infection may recrudess $^{1}$ with subsequent neutropenic episodes. Surgical resection of fungal disease, if possible, ${ }^{14}$ is particularly important, together with a regimen of antifungal treatment (amphotericin B with flucytosine has been used $^{2}$ with some success).

Itraconazole has great potential as a drug for prophylaxis against the reactivation of fungal infection in patients with treated invasive fungal disease who need to undergo further immunosuppressive chemotherapy to achieve or consolidate remission. Its low toxicity, administration by mouth, and good activity against aspergillus make it attractive for use during subsequent periods of immunosuppression. An operation is not always possible, either due to the extent of the infection or to the clinical status of the patient. Renal toxicity is of importance in patients who have already received large cumulative doses of amphotericin B and these patients will almost certainly need supportive care with nephrotoxic drugs. The availability of a prophylactic drug with no renal toxicity is therefore useful. Patients who have undergone intensive chemotherapy or bone marrow transplantation will need to continue to receive antifungal drugs for up to six months after the end of treatment.

1 Robertson MJ, Larson RA. Recurrent fungal pneumonias in patients with acute nonlymphocytic leukemia undergoing multiple courses of intensive chemotherapy. $A m \mathcal{F} M e d$ 1988; 84: 233-9.

2 Karp JE, Burch PA, Merz WG. An approach to intensive antileukemia therapy in patients with previous invasive Aspergillosis. Am $\mathcal{F}$ Med 1988; 85: 203-6.

3 Tollemar J, Ringden O, Bostrom L, Nilsson B, Sunberg B. Variables predicting deep fungal infections in bone marrow transplant recipients. Bone Marrow Transplant 1989; 4: 635-41.

4 Gerson SL, Talbot GH, Lusk E, Hurwitz S, Strom BL, Cassileth PA. Invasive pulmonary aspergillosis in adult acute leukemia: clinical clues to its diagnosis. $\mathcal{F}$ Clin Oncol 1985; 3: 1109-16.

5 Caillot D, Kisterman JP, Solary E, et al. Itraconazole (ITZ) in leukemic patients with invasive pulmonary aspergillosis (IPA) pretreated by amphotericin B (AmB). Proceedings of Interscience Conference on Antimicrobial Agents and Chemotherapy (ICAAC) 1990; 946: 244.

6 Milliken ST, Powles RI. Antifungal prophylaxis in bone marrow transplantation. Rev Infect Dis 1990; 12: S374-9.

7 Tricot G, Joosten E, Boogaerts MA, Vande Pitte J, Cauwenbergh G. Ketoconazole vs. itraconazole for antifungal prophylaxis in patients with severe granulocytofungal prophylaxis in patients with severe granulocytopenia: preliminary results of two
Rev Infect Dis 1987; 9: S94-9.

8 Degregorio MW, Lee WMF, Linker CA, Jacobs RA, Reis $\mathrm{CA}$. Fungal infections in patients with acute leukemia. Am f Med 1982; 73: 543-8.

9 Boogaerts MA, Verhoef GE, Zachee P,. Demuynck H, Verbist L, De Beule K. Antifungal prophylaxis with itraconazole in prolonged neutropenia: correlation with plasma levels. Mycoses 1989; 32: 103-8.

10 Thunnissen PLM, Sizoo W, Hendriks WDH. Safety and efficacy of itraconazole in prevention of fungal infections in neutropenic patients. Neth $\mathcal{f}$ Med 1991; 39: tions in

11 Burch PA, Karp JE, Merz WG, Kuhlman JE, Fishman EK. Favourable outcome of invasive aspergillosis in patients with acute leukemia. F Clin Oncol 1993; 5: 1985-7.

12 Graham Watson J. Problems of infection after bone marrow transplantation. $\mathcal{F}$ Clin Pathol 1983; 36: 683-92.

13 Lupinetti FM, Behrendt DM, Giller RH, Trigg ME, de Alarcon P. Pulmonary resection for fungal infection in children undergoing bone marrow transplantation. f Thorac Cardiovasc Surg 1992; 104: 684-7.

14 Terrell CL, Hughes CE. Antifungal agents used for deepseated mycotic infections. Mayo Clin Proc 1992; 67: 69-91.

15 Negroni R, Palmieri O, Koren F, Tiraboschi IN, Galimberti RK. Oral treatment of paracoccidioidomycosis and histoplasmosis with itraconazole in humans. Rev Infect Dis plasmosis with itrac

16 Cauwenbergh G, De Doncker P, Stoops K, De Dier A-M, Goyvaerts H, Schuermans V. Itraconazole in the treatment of human mycoses: review of three years of clinical experience. Rev Infect Dis 1987; 9: S146-52

17 De Beule K, De Doncker P, Cauwenbergh G, et al. The treatment of aspergillosis and aspergilloma with itraconazole, clinical results of an open international study (1982-1987). Mycoses 1988; 31: 476-85.

18 Cauwenbergh $\mathrm{G}$. Treatment of aspergillosis: itraconazole a break-through? Proceedings of the XX Scientific Meeting of the German Mycological Society. May 1986, Meeting of

19 Buckner CD, Clift RA, Sanders JE, et al. Protective environment for marrow transplant recipients. A prospective study. Ann Intern Med 1978; 89: 893-901. 